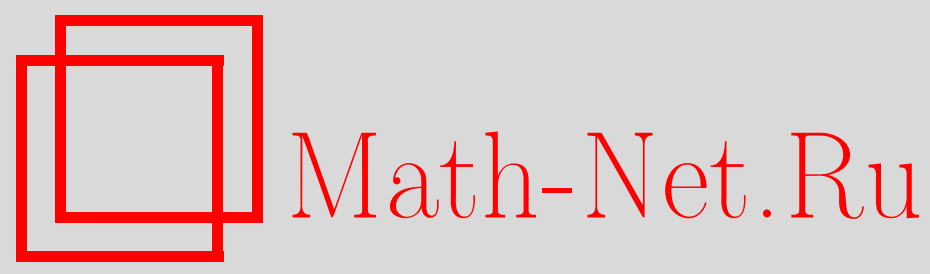

В. В. Лысиков, Об алгебрах почти минимального ранга, Дискрет. матем., 2012, том 24, выпуск 4, 3-18

DOI: https://doi.org/10.4213/dm1206

Использование Общероссийского математического портала Math-Net.Ru подразумевает, что вы прочитали и согласны с пользовательским соглашением http://www.mathnet.ru/rus/agreement

Параметры загрузки:

IP: 3.80 .181 .102

26 апреля 2023 г., 02:44:46 


\title{
Об алгебрах почти минимального ранга
}

\author{
() 2012 г. В. В. Лысиков
}

\begin{abstract}
В работе рассматривается билинейная сложность умножения в локальных и полупростых алгебрах над бесконечным полем характеристики, отличной от 2. Получен критерий почти минимальности ранга локальной алгебры. Определено значение билинейной сложности для алгебр обобщенных кватернионов над таким полем, доказано, что любая простая алгебра почти минимального ранга является алгеброй обобщенных кватернионов. Этот результат используется для классификации полупростых алгебр почти минимального ранга.

Работа выполнена при финансовой поддержке Российского фонда фундаментальных исследований, проект 12-01-91331-ННИО-а.
\end{abstract}

\section{1. Введение}

Одним из важнейших объектов алгебраической теории сложности вычислений являются билинейные алгоритмы, изучение которых началось в связи с одной из основных задач этой теории - задачи определения сложности умножения матриц. Помимо умножения матриц, часто рассматриваемым классом билинейных отображений является умножение в некотором классе алгебр, в частности, в ассоциативных алгебрах с единицей, к которым относится и алгебра квадратных матриц.

Важным результатом о сложности умножения в ассоциативных алгебрах является нижняя оценка, полученная Алдером и Штрассеном [1]. После получения этой оценки в ряде работ рассматривался вопрос об описании всех алгебр минимального ранга, то есть тех, для которых оценка Алдера-Штрассена для ранга является точной. Окончательное описание всех алгебр минимального ранга было получено М. Блезером в [2], а в [3] было начато изучение алгебр, ранг которых на 1 больше оценки Алдера-Штрассена. В частности, в [3] получено полное описание полупростых алгебр над полем $\mathbf{R}$, обладающих этим свойством. Мы обобщим этот результат на случай произвольного бесконечного поля характеристики, отличной от 2.

\section{2. Основные понятия. Модель вычислений}

Приведем определения основных понятий, связанных с билинейными отображениями.

Определение 1. Пусть $F$ - поле, и $U, V, W$ - линейные пространства над ним. Отображение $\varphi: U \times V \rightarrow W$ называется билинейным, если оно линейно по каждому аргументу, 
то есть

$$
\begin{aligned}
& \varphi(\lambda x+\mu y, z)=\lambda \varphi(x, z)+\mu \varphi(y, z), \\
& \varphi(x, \lambda y+\mu z)=\lambda \varphi(x, y)+\mu \varphi(x, z) .
\end{aligned}
$$

Определение 2. Алгеброй над полем $F$ называется линейное пространство $A$, снабженное билинейным отображением $: A \times A \rightarrow A$, называемым умножением. Алгебра называется ассоциативной, если выполняется свойство ассоциативности умножения:

$$
a(b c)=(a b) c
$$

для любых $a, b, c \in A$.

Единицей в алгебре $A$ называется такой элемент $1 \in A$, что

$$
1 \cdot a=a \cdot 1=a
$$

для любого $a \in A$.

Замечание 1. В данной работе мы будем рассматривать только конечномерные ассоциативные алгебры с единицей. В дальнейшем термин алгебра будет обозначать только такие алгебры. Если $A-$ алгебра с единицей, то существует вложение $F \rightarrow A$, ставящее в соответствие скаляру $\alpha$ элемент алгебры $\alpha \cdot 1$. Мы будем иногда отождествлять $F$ с образом этого вложения.

Для изучения сложности билинейных отображений используются модели билинейных и квадратичных алгоритмов.

Определение 3. Билинейным алгоритмом сложности $r$ для $\varphi$ называется набор троек $\left(f_{1}, g_{1}, z_{1} ; \ldots ; f_{r}, g_{r}, z_{r}\right), f_{k} \in U^{*}, g_{k} \in V^{*}, z_{k} \in W$, такой, что для любых $u \in U, v \in V$ выполнено соотношение

$$
\varphi(u, v)=\sum_{k=1}^{r} f_{k}(u) g_{k}(v) z_{k} .
$$

Минимально возможная сложность билинейного алгоритма называется билинейной сложностью или рангом отображения $\varphi$ и обозначается $R(\varphi)$. Рангом алгебры будем называть ранг умножения в ней.

В данной работе рассматриваются только билинейные алгоритмы. Некоторые используемые результаты, изначально сформулированные для более сильной модели квадратичных алгоритмов, мы приводим в измененной формулировке.

Понятие ранга билинейного отображения допускает удобную интерпретацию в терминах мультилинейной алгебры. Каждому билинейному отображению $\varphi: U \times V \rightarrow W$ естественным образом соответствует тензор из пространства $U^{*} \otimes V^{*} \otimes W$, а билинейные алгоритмы соответствуют представлениям этого тензора в виде суммы разложимых тензоров $f_{k} \otimes g_{k} \otimes z_{k}$. В связи с этим мы будем записывать билинейные алгоритмы следующим образом:

$$
\varphi=\sum_{k} f_{k} \otimes g_{k} \otimes z_{k}
$$

Введем некоторые обозначения. Символом 0 мы будем обозначать нулевое подпространство в любом линейном пространстве. Линейную оболочку множества векторов $M$ будем обозначать $\operatorname{lin}(M)$. Символом $[a, b]$ будем обозначать коммутатор двух матриц или элементов алгебры:

$$
[a, b]=a b-b a .
$$




\section{3. Структура алгебр}

В этом разделе мы приводим используемые далее факты о структуре конечномерных алгебр над полем. Эти результаты являются классическими и изложены во многих монографиях и учебных пособиях, например, в [4] и [5].

Определение 4. Подпространство $L$ алгебры $A$ называется левым идеалом, если оно инвариантно относительно умножения на любой элемент алгебры слева:

$$
a x \in L
$$

для любых $a \in A, x \in L$.

Аналогично определяются правые идеалы.

Подпространство называется двусторонним идеалом, или просто идеалом, если оно является левым и правым идеалом.

Идеал называется собственным, если он не равен нулевому идеалу $\mathbf{0}$ и не совпадает со всей алгеброй $A$.

Собственный или нулевой идеал называется максимальным, если он не содержится ни в каком другом собственном идеале.

Определение 5. Идеал называется нильпотентным, если для некоторого натурального $n$ произведение любых $n$ элементов идеала равно 0.

Алгебра называется полупростой, если она не имеет нильпотентных идеалов, кроме $\mathbf{0 .}$

Алгебра называется простой, если она не имеет собственных идеалов.

Алгебра называется локальной, если она имеет единственный максимальный левый идеал.

Определение 6. Прямым произведением алгебр $A$ и $B$ называется алгебра $A \times B$, состоящая из пар вида $(a, b)$, где $a \in A, b \in B$, с покоординатным умножением.

Определение 7. Элемент $a$ алгебры $A$ называется обратимым, если существует элемент $a^{-1} \in A$ такой, что

$$
a a^{-1}=a^{-1} a=1 .
$$

Алгебра называется алгеброй с делением, если любой ее ненулевой элемент обратим.

Следующая теорема известна как теорема Веддерберна-Артина (см. теорему 4.3 в [5]).

Теорема 1. Алгебра полупроста тогда и только тогда, когда она является прямым произведением простых алгебр. Простыми алгебрами являются алгебры матрии над алгебрами с делением, и только они.

Следующая теорема есть теорема 2.2 в [5].

Теорема 2. Локальная алгебра имеет единственный максимальный идеал, совпадающий с максимальным левым идеалом. Элемент локальной алгебры обратим тогда и только тогда, когда он не содержится в максимальном идеале.

Определение 8. Центром алгебры $A$ называется подалгебра, состоящая из элементов, коммутирующих с любым элементом алгебры:

$$
C(A)=\{x \mid x a=a x \text { для любого } a \in A\} .
$$

Алгебра над $F$ называется центральной, если ее центр совпадает с $F$. 
Следующая теорема есть теорема 5.1 в [5].

Теорема 3. Любая простая алгебра является простой алгеброй над своим иентром. Размерность простой центральной алгебры является квадратом.

Следствие 1. Не сущзествует некоммутативных простых алгебр, размерность которых является свободным от квадратов числом.

Определение 9. Алгеброй обобщенных кватернионов над $F$ называется простая центральная алгебра размерности 4.

Теорема 4 ([6]). Если $\operatorname{char} F \neq 2$, то любая алгебра обобщенньх кватернионов над $F$ порождается двумя элементами $i$ u , удовлетворяющими условиям

$$
\begin{aligned}
& i^{2}=p, \\
& j^{2}=q, \\
& i j=-j i,
\end{aligned}
$$

где $p, q \in F-$ некоторые ненулевые элементы. Элементы $1, i, j, k=i j$ образуют базис алгебры. Такую алгебру будем обозначать $\left(\frac{F}{p, q}\right)$.

Теорема 5 ([6]). Любая алгебра обобщенных кватернионов $H$ над полем $F$ либо изоморфна алгебре матрии $F^{2 \times 2}$, либо является некоммутативной алгеброй с делением. Любая некоммутативная алгебра с делением размерности 4 является алгеброй обобщенных кватернионов.

Также нам потребуются результаты о структуре расширений полей простой характеристики.

Определение 10. Многочлен степени $n$ над полем $F$ называется сепарабельным, если все $n$ его корней, принадлежащих алгебраическому замыканию $\bar{F}$, различны. Элемент некоторого расширения поля $F$ называется сепарабельным, если его минимальный многочлен сепарабелен. Расширение называется сепарабельным, если все его элементы сепарабельны, и чисто несепарабельным, если все его элементы, не принадлежащие $F$, несепарабельны.

Определение 11. Расширение поля $F$ называется простым, если оно получается присоединением к $F$ одного элемента.

Теорема 6 ([4], §44). Любое расширение поля характеристики 0 сепарабельно. Любое расширение $K$ поля $F$ простой характеристики р является чисто несепарабельным расширением некоторого сепарабельного расширения $E$.

Следующая теорема о примитивном элементе доказана в §46 в [4].

Теорема 7. Конечномерное сепарабельное расширение является простым. Расширение, полученное присоединением одного несепарабельного элемента к сепарабельному расширению, является простьм.

Теорема 8 ([4], §44). Минимальный многочлен любого элемента чисто несепарабельного расширения $K$ поля $E$ простой характеристики $p$ имеет вид $x^{p^{e}}-c$ для некоторых $e \in \mathbf{N}, c \in E$.

Следствие 2. Размерность несепарабельного расширения $K$ поля $F$ простой характеристики р имеет вид $\mathrm{np}^{f}$. Размерность любого простого подрасширения $K$ имеет вид $m p^{e}$, где $m \leqslant n$, $e \leqslant f$, причем е $<f$, если $K$ не является простыл. 


\section{4. Методы получения нижних оценок}

Основным методом получения нижних оценок в алгебраической теории сложности является метод подстановки, который для билинейных отображений может быть сформулирован в виде леммы о разделении. С использованием этого метода А. Алдер и Ф. Штрассен доказали следующую общую оценку для сложности умножения в алгебрах.

Теорема 9. Пусть $A, B$ - алгебры. Ранг прямого произведения $A \times B$ оиенивается снизу следующим образом:

$$
R(A \times B) \geqslant 2 \operatorname{dim} A-t(A)+R(B),
$$

где $t(A)$ - число максимальных идеалов $A$.

В частности, если $B=\mathbf{0}$, то

$$
R(A) \geqslant 2 \operatorname{dim} A-t(A) .
$$

Для алгебр с делением известен следующий более сильный результат Баура (теорема 17.27 в [7]).

Теорема 10. Пусть $D$ - алгебра с делением размерности $n$ над полем $F$. Тогда

$$
R(D) \geqslant 2 n-2+\frac{n}{\operatorname{dim} F(a)}
$$

для некоторого элемента $a \in D$.

Частным случаем применения метода подстановки является ограничение одного из аргументов на подпространство малой размерности. Сформулируем леммы, позволяющие использовать этот прием.

Определение 12. Левым ядром $\operatorname{lker} \varphi$ билинейного отображения $\varphi: U \times V \rightarrow W$ называется множество всех таких $u \in U$, что $\varphi(u, v)=0$ для любого $v \in V$. Аналогично определяется правое ядро rker $\varphi$.

Лемма 1. Пусть $\varphi: U \times V \rightarrow W-$ билинейное отображение. Если lker $\varphi=\mathbf{0}$, то в любом билинейном алгоритме $\sum_{k=1}^{r} f_{k} \otimes g_{k} \otimes z_{k}$ для $\varphi$ функциональл $f_{k}$ порождают в линейной оболочке все пространство $U^{*}$.

Доказательство. Проведем доказательство от противного. Если $\operatorname{lin}\left(\left\{f_{1}, \ldots, f_{r}\right\}\right) \subsetneq U^{*}$, то существует ненулевой элемент $u \in U$ такой, что

$$
f_{1}(u)=\ldots=f_{r}(u)=0 .
$$

Из определения билинейного алгоритма следует, что $\varphi(u, v)=0$ для любого $v \in V$, то есть $u \in \operatorname{lker} \varphi$.

Заметим, что условие доказанной леммы верно, если $\varphi-$ умножение в некоторой алгебре с единицей.

Лемма 2. Пусть $\varphi: U \times V \rightarrow W-$ билинейное отображение, $\sum_{k=1}^{r} f_{k} \otimes g_{k} \otimes z_{k}-$ алгоритм для $\varphi$, а $U^{\prime}-$ подпространство $U$. Тогда

$$
r \geqslant q+R\left(\left.\varphi\right|_{U^{\prime} \times V}\right)
$$

где q - число $f_{k}$, равных тождественно нулю на $U^{\prime}$. 
Доказательство. Ограничив все функционалы $f_{k}$ на подпространство $U^{\prime}$, мы получим алгоритм $\left.\sum_{k=1}^{r} f_{k}\right|_{U^{\prime}} \otimes g_{k} \otimes z_{k}$ для $\left.\varphi\right|_{U^{\prime} \times V}$. В нем можно опустить слагаемые, для которых $\left.f_{k}\right|_{U^{\prime}}=0$, после чего получится алгоритм для $\left.\varphi\right|_{U^{\prime} \times V}$ сложности $r-q$, существование которого означает, что $R\left(\left.\varphi\right|_{U^{\prime} \times V}\right) \leqslant r-q$.

Для оценки ранга отображений с малой размерностью $U^{\prime}$ можно использовать следующий результат, принадлежащий Штрассену (см. [8]).

Теорема 11. Пусть $\varphi: F^{3} \times U \rightarrow U-$ билинейное отображение, $e_{1}, e_{2}, e_{3}-$ базис $F^{3}$, и операторы $A_{i}$ определяются соотношением

$$
A_{i} x=\varphi\left(e_{i}, x\right),
$$

причем $A_{1}$ невырожден. Тогда

$$
R(\varphi) \geqslant \operatorname{dim} U+\frac{1}{2} \operatorname{rk}\left[A_{1}^{-1} A_{2}, A_{1}^{-1} A_{3}\right] .
$$

С использованием этого метода в [9] были доказаны оценки ранга алгебр, приведенные в следующей теореме.

Теорема 12. Для некоммутативной алгебры с делением D справедлива оценка

$$
R(D) \geqslant \frac{5}{2} \operatorname{dim} D-3 \text {. }
$$

Теорема 13. Пусть $D$ - алгебра с делением, $n \geqslant 2, A \cong D^{n \times n}-$ простая алгебра. Тогда

$$
R(A) \geqslant \frac{5}{2} \operatorname{dim} A-3 n .
$$

\section{5. Полупростые алгебры почти минимального ранга}

Определение 13. Алгебра $A$ называется алгеброй минимального ранга, если для ее ранга достигается оценка Алдера-Штрассена, то есть

$$
R(A)=2 \operatorname{dim} A-t(A),
$$

и алгеброй почти минимального ранга, если ее ранг на 1 больше этой величины.

Введем обозначение

$$
\Delta(A)=R(A)-2 \operatorname{dim} A+t(A) .
$$

Из теоремы Алдера-Штрассена следует, что

$$
\Delta(A \times B) \geqslant \Delta(A),
$$

поскольку $t(A \times B) \geqslant t(A)+t(B)$ : для любого максимального идеала $I \subset A(J \subset B)$ пространство $I \times B(A \times J)$ является максимальным идеалом $A \times B$.

Из (16), в свою очередь, следует справедливость следующего утверждения.

Предложение 1. Пусть

$$
A \cong A_{1} \times A_{2} \times \ldots \times A_{t}
$$

есть разложение полупростой алгебры в произведение простых алгебр. Если А имеет почти минимальный ранг, то все $A_{i}$ являются алгебрами минимального или почти минимального ранга. 
Алгебры минимального ранга были описаны в [2]. Для простых алгебр эта классификация дает следующий результат.

Теорема 14. Простыми алгебрами минимального ранга над полем $F$ являются алгебра матрии $F^{2 \times 2}$ и простые расширения $K$ поля $F$, причем для случая конечного $F$ должно выполняться соотношение

$$
2 \operatorname{dim}_{F} K-2 \leqslant \# F .
$$

В частности, некоммутативные алгебры с делением не могут быть алгебрами минимального ранга.

Следствие 3. Если D - некоммутативная алгебра с делением, то

$$
R(D) \geqslant 2 \operatorname{dim} D .
$$

В [3] были описаны полупростые алгебры над полем действительных чисел $\mathbf{R}$. Многие результаты этой работы верны и для произвольного поля.

Рассмотрим простую алгебру $A \cong D^{n \times n}$, где $D-$ алгебра с делением, $\operatorname{dim} D=d$. Если $n \geqslant 4$, то $A$ не может быть алгеброй почти минимального ранга по теореме 13 . То же можно сказать и для случаев $n=3, d \geqslant 3$ и $n=2, d \geqslant 4$. Если $n=2$ и $d=1$, то $A \cong F^{2 \times 2}-$ алгебра минимального ранга.

При $n=1$ возможны три существенно различных случая: некоммутативная алгебра с делением $D$, простое расширение поля $F$, и непростое, а потому несепарабельное, расширение. Простые расширения являются алгебрами минимального ранга. Для непростого расширения мы применим оценку теоремы 10 . Пусть $F$ - поле простой характеристики $p>2, K-$ его непростое несепарабельное расширение, $\operatorname{dim} K=n=n^{\prime} p^{f}$. Из результатов о строении несепарабельных расширений, приведенных в разделе 3 , следует, что размерность любого простого подполя $F(a)$ будет равна $m=m^{\prime} p^{e}$ для некоторых $m^{\prime} \leqslant n^{\prime}$ и $e<f$. Справедливы неравенства

$$
\begin{aligned}
R(K) & \geqslant 2 n-2+\frac{n}{m} \\
& \geqslant 2 n-2+p>2 n,
\end{aligned}
$$

откуда следует, что $K$ не может быть алгеброй почти минимального ранга.

Некоммутативные алгебры с делением не являются алгебрами почти минимального ранга при $d \geqslant 8$ по теореме 12 . Так как, по следствию из теоремы 3 , не существует некоммутативных алгебр с делением размерностей 2, 3, 5, 6 и 7, остается рассмотреть следующие случаи:

$$
\begin{array}{ll}
n=1, & d=4 ; \\
n=2, & d \in\{2,3\} ; \\
n=3, & d \in\{1,2\} .
\end{array}
$$

Случай $n=1, d=4$, то есть алгебры кватернионов с делением, мы рассмотрим в разделе 7. Случай $n=2, d=2$ разобран в [3] для $F=\mathbf{R}$. В предисловии авторы этой работы отмечают, что их оценка верна и для произвольного поля $F$. Для интересующих нас полей с char $F \neq 2$ доказательство теоремы 3 из [3] может быть дословно повторено с заменой $\mathbf{R}$ на $F, \mathbf{C}$ на произвольное квадратичное расширение $K \cong F[i] /\left(i^{2}-p\right)$ для некоторого $p$, не являющегося квадратом, и соответствующим образом определенной билинейной формой $\langle\cdot, \cdot\rangle$ на $K^{2 \times 2}$. Таким образом, можно сформулировать эту теорему в следующем виде. 
Теорема 15. Пусть $K-$ квадратичное расширение поля $F$. Тогда

$$
R_{F}\left(K^{2 \times 2}\right) \geqslant 17 \text {. }
$$

Случай $n=3, d=1$ разобран в [10], где доказана следующая оценка.

Теорема 16. Ранг алгебры матрии размера $3 \times 3$ удовлетворяет неравенству

$$
R\left(F^{3 \times 3}\right) \geqslant 19 .
$$

Оставшиеся два случая мы рассмотрим в разделе 8 .

\section{6. Билинейные отображения, ранг которых равен сумме двух размерностей}

Введем обозначения

$$
\operatorname{dim} U=m, \quad \operatorname{dim} V=n .
$$

Прежде чем обратиться к сложности умножения кватернионов, мы докажем некоторые общие утверждения о билинейных алгоритмах сложности $m+n$ для некоторого довольно широкого класса билинейных отображений.

Определение 14. Назовем элемент $u_{0} \in U$ (левым) $\varphi$-регулярным, если линейный оператор $\varphi\left(u_{0}, \cdot\right)$ инъективен, то есть $\varphi\left(u_{0}, v\right)=0$ тогда и только тогда, когда $v=0$.

Определение 15. Назовем билинейный алгоритм

$$
\varphi=\sum_{k=1}^{r} f_{k} \otimes g_{k} \otimes z_{k}
$$

двухкомпонентным, если множество индексов $\{1, \ldots, r\}$ можно разбить на непересекающиеся множества $I$ и $J$ такие, что $\left\{f_{i} \mid i \in I\right\}$ и $\left\{g_{j} \mid j \in J\right\}$ являются базисами пространств $U^{*}$ и $V^{*}$ соответственно.

В терминах разделения подпространств (см. [7]) двухкомпонентные алгоритмы можно описать как алгоритмы ранга $m+n$, разделяющие $(U, V, \boldsymbol{0})$.

Лемма 3. Если

$$
R(\varphi)=m+n, \quad \operatorname{lker} \varphi=\mathbf{0},
$$

и в любом базисе пространства U найдется $\varphi$-регулярный элемент, то любой оптимальный билинейный алгоритм для $\varphi$ является двухкомпонентным.

Доказательство. Так как $\operatorname{lker} \varphi=\mathbf{0}$, то функционалы $f_{1}, \ldots, f_{m+n}$ порождают все пространство $U^{*}$. Пусть, без ограничения общности, $\left(f_{1}, \ldots, f_{m}\right)-$ базис $U^{*}$, а элемент $u_{1}$ двойственного базиса $\left(u_{1}, \ldots, u_{m}\right)$ является $\varphi$-регулярным. Из $\varphi$-регулярности $u_{1}$ следует, что функционалы $g_{1}, g_{m+1}, \ldots, g_{m+n}$ порождают все пространство $V^{*}$, так как иначе, взяв ненулевой элемент $v \in \operatorname{ker} g_{1} \cap \bigcap_{k=m+1}^{m+n} \operatorname{ker} g_{k}$, мы получим, что $\varphi\left(u_{1}, v\right)=0$. 
Введем обозначение

$$
G=\operatorname{lin}\left(\left\{g_{m+1}, \ldots, g_{m+n}\right\}\right) .
$$

Если $G=V^{*}$, то множества индексов $I=\{1, \ldots, m\}$ и $J=\{m+1, \ldots, m+n\}$ образуют требуемое разбиение. В противном случае $\operatorname{dim} G=n-1$, и существует единственная, с точностью до умножения на константу, линейная зависимость между $g_{m+1}, \ldots, g_{m+n}$. Пусть, без ограничения общности, $g_{m+1}, \ldots, g_{m+s}$ входят в уравнение линейной зависимости с ненулевыми коэффициентами, а остальные функционалы - с нулевыми.

Если найдется $f_{p} \mathrm{c} m+1 \leqslant p \leqslant m+s$ такой, что в разложении его по базису $f_{1}, \ldots, f_{m}$ присутствует с ненулевым коэффициентом $f_{1}$, то множества индексов $I=\{2, \ldots, m, p\}$ и $J=\{1, m+1, \ldots, m+n\} \backslash\{p\}$ образуют требуемое разбиение. Иначе, взяв ненулевой элемент $v_{0} \in V$ такой, что $g_{1}\left(v_{0}\right)=g_{m+s+1}\left(v_{0}\right)=\ldots=g_{m+n}\left(v_{0}\right)=0$, получим, что $\varphi\left(u_{1}, v_{0}\right)=0$, поскольку $f_{k}\left(u_{1}\right)=0$ при $2 \leqslant k \leqslant m$ по определению $u_{1}$ и при $m+1 \leqslant$ $k \leqslant m+s$, так как все $f_{k}$ с индексами из этого промежутка не содержат $f_{1}$ в разложении по базису $\left(f_{1}, \ldots, f_{m}\right)$. Получаем противоречие с $\varphi$-регулярностью элемента $u_{1}$.

Лемма 4. Двухкомпонентный билинейный алгоритм для ф сущзествует тогда и только тогда, когда существуют базисы $u_{1}, \ldots, u_{m} u v_{1}, \ldots, v_{n}$ пространств $U$ u $V$ соответственно, и наборы $z_{1}^{\prime}, \ldots, z_{m}^{\prime} u z_{1}^{\prime \prime}, \ldots, z_{n}^{\prime \prime}$ элементов $W$, удовлетворяющие условию $\varphi\left(u_{i}, v_{j}\right) \in \operatorname{lin}\left(\left\{z_{i}^{\prime}, z_{j}^{\prime \prime}\right\}\right)$.

Доказательство. Пусть существует двухкомпонентный билинейный алгоритм для $\varphi$ :

$$
\varphi=\sum_{i=1}^{m} f_{i} \otimes G_{i} \otimes z_{i}^{\prime}+\sum_{j=1}^{n} F_{j} \otimes g_{j} \otimes z_{j}^{\prime \prime},
$$

где $f_{1}, \ldots, f_{m}$ и $g_{1}, \ldots, g_{n}-$ базисы соответствующих пространств. Пусть $u_{1}, \ldots, u_{m}$ и $v_{1}, \ldots, v_{n}-$ двойственные им базисы. Тогда

$$
\varphi\left(u_{i}, v_{j}\right)=G_{i}\left(v_{j}\right) z_{i}^{\prime}+F_{j}\left(u_{i}\right) z_{j}^{\prime \prime} .
$$

Обратно, если существуют базисы $\left(u_{i}\right),\left(v_{j}\right)$ и наборы $\left(z_{i}^{\prime}\right),\left(z_{j}^{\prime \prime}\right)$ такие, что

$$
\varphi\left(u_{i}, v_{j}\right)=\lambda_{i j} z_{i}^{\prime}+\mu_{i j} z_{j}^{\prime \prime}
$$

a $\left(f_{i}\right)$ и $\left(g_{j}\right)$ - базисы, двойственные к $\left(u_{i}\right)$ и $\left(v_{j}\right)$ соответственно, то билинейный алгоритм

$$
\sum_{i=1}^{m} f_{i} \otimes\left(\sum_{j=1}^{n} \lambda_{i j} g_{j}\right) \otimes z_{i}^{\prime}+\sum_{j=1}^{n}\left(\sum_{i=1}^{m} \mu_{i j} f_{i}\right) \otimes g_{j} \otimes z_{j}^{\prime \prime}
$$

вычисляет $\varphi$. Чтобы убедиться в этом, в силу билинейности, достаточно проверить равенство (4) на парах $\left(u_{i}, v_{j}\right)$ элементов базисов.

В случае, когда $\varphi$ - умножение в алгебре, $\varphi$-регулярные элементы есть обратимые элементы алгебры. По теореме 2, в локальной алгебре обратимы те и только те элементы, которые не лежат в единственном максимальном идеале (в частности, в алгебре с делением обратимы все элементы), так что любой базис локальной алгебры содержит обратимый элемент. Используя этот факт и доказанные выше леммы, мы можем связать существование алгоритмов ранга $2 \operatorname{dim} A$ с существованием базисов, обладающих определенными свойствами. 
Следствие 4. Пусть $A-$ локальная алгебра, $\operatorname{dim} A=n$. Если $A$ не является алгеброй минимального ранга, то есть $R(A) \geqslant 2 n$, то $A$ имеет почти минимальный ранг тогда и только тогда, когда в А существует пара базисов $u_{1}=1, u_{2}, \ldots, u_{n} u v_{1}=1, v_{2}, \ldots, v_{n}$ и пара наборов элементов $z_{1}^{\prime}, \ldots, z_{n}^{\prime} u z_{1}^{\prime \prime}, \ldots, z_{n}^{\prime \prime}$ такие, что

$$
u_{i} v_{j} \in \operatorname{lin}\left(\left\{z_{i}^{\prime}, z_{j}^{\prime \prime}\right\}\right) .
$$

Доказательство. Утверждение следует из лемм 1 и 2. Элементы $u_{1}$ и $v_{1}$ можно взять равными 1 , так как одновременная перестановка элементов $\left(u_{i}\right)$ и $\left(z_{i}^{\prime}\right)$ или $\left(v_{j}\right)$ и $\left(z_{j}^{\prime \prime}\right)$ и преобразование $u \mapsto u_{1}^{-1} u, v \mapsto v v_{1}^{-1}, z \mapsto u_{1}^{-1} z v_{1}^{-1}$ не влияют на выполнение соотношения (23).

\section{7. Сложность умножения обобщенных кватернионов}

Пусть $F$ - поле, характеристика которого отлична от 2, а $H=\left(\frac{F}{p, q}\right)-$ некоторая алгебра обобщенных кватернионов с делением. Так как $H$ - некоммутативная алгебра с делением, она не может быть алгеброй минимального ранга, то есть $R(H) \geqslant 8$. В силу результатов предыдущего раздела, для доказательства того, что $R(H)=8$, достаточно привести пример пары базисов $\left(u_{i}\right),\left(v_{j}\right)$ и пары наборов $\left(z_{i}^{\prime}\right),\left(z_{j}^{\prime \prime}\right)$, удовлетворяющих условию (23).

Возьмем в качестве $\left(u_{i}\right)$ и $\left(v_{j}\right)$ стандартный кватернионный базис

$$
\left(u_{1}, u_{2}, u_{3}, u_{4}\right)=\left(v_{1}, v_{2}, v_{3}, v_{4}\right)=(1, i, j, k),
$$

а наборы $z_{i}^{\prime}$ и $z_{j}^{\prime \prime}$ определим следующим образом:

$$
\begin{aligned}
&\left(z_{1}^{\prime}, z_{2}^{\prime}, z_{3}^{\prime}, z_{4}^{\prime}\right)=(1+\alpha i+\beta j+\gamma k, 1+\alpha i-\beta j-\gamma k, \\
&1-\alpha i+\beta j-\gamma k, 1-\alpha i-\beta j+\gamma k), \\
&\left(z_{1}^{\prime \prime}, z_{2}^{\prime \prime}, z_{3}^{\prime \prime}, z_{4}^{\prime \prime}\right)=(1-\alpha i-\beta j-\gamma k, 1-\alpha i+\beta j+\gamma k, \\
&1+\alpha i-\beta j+\gamma k, 1+\alpha i+\beta j-\gamma k),
\end{aligned}
$$

где $\alpha, \beta, \gamma$ - некоторые ненулевые константы из $F$. Непосредственно проверяется, что условие (23) для указанных кватернионов выполняется со следующими коэффициентами:

\begin{tabular}{c|cccc} 
& $v_{1}$ & $v_{2}$ & $v_{3}$ & $v_{4}$ \\
\hline$u_{1}$ & $\frac{1}{2} z_{1}^{\prime}+\frac{1}{2} z_{1}^{\prime \prime}$ & $\frac{1}{2 \alpha} z_{1}^{\prime}-\frac{1}{2 \alpha} z_{2}^{\prime \prime}$ & $\frac{1}{2 \beta} z_{1}^{\prime}-\frac{1}{2 \beta} z_{3}^{\prime \prime}$ & $\frac{1}{2 \gamma} z_{1}^{\prime}-\frac{1}{2 \gamma} z_{4}^{\prime \prime}$ \\
$u_{2}$ & $\frac{1}{2 \alpha} z_{2}^{\prime}-\frac{1}{2 \alpha} z_{1}^{\prime \prime}$ & $\frac{p}{2} z_{2}^{\prime}+\frac{p}{2} z_{2}^{\prime \prime}$ & $-\frac{1}{2 \gamma} z_{2}^{\prime}+\frac{1}{2 \gamma} z_{3}^{\prime \prime}$ & $-\frac{p}{2 \beta} z_{2}^{\prime}+\frac{p}{2 \beta} z_{4}^{\prime \prime}$ \\
$u_{3}$ & $\frac{1}{2 \beta} z_{3}^{\prime}-\frac{1}{2 \beta} z_{1}^{\prime \prime}$ & $\frac{1}{2 \gamma} z_{3}^{\prime}-\frac{1}{2 \gamma} z_{2}^{\prime \prime}$ & $\frac{q}{2} z_{3}^{\prime}+\frac{q}{2} z_{3}^{\prime \prime}$ & $\frac{q}{2 \alpha} z_{3}^{\prime}-\frac{q}{2 \alpha} z_{4}^{\prime \prime}$ \\
$u_{4}$ & $\frac{1}{2 \gamma} z_{4}^{\prime}-\frac{1}{2 \gamma} z_{1}^{\prime \prime}$ & $\frac{p}{2 \beta} z_{4}^{\prime}-\frac{p}{2 \beta} z_{2}^{\prime \prime}$ & $-\frac{q}{2 \alpha} z_{4}^{\prime}+\frac{q}{2 \alpha} z_{3}^{\prime \prime}$ & $-\frac{p q}{2} z_{4}^{\prime}-\frac{p q}{2} z_{4}^{\prime \prime}$
\end{tabular}

Учитывая нижнюю оценку $R(H) \geqslant 8$, получаем, что 8 является точным значением ранга $H$.

Теорема 17. Пусть $F-$ поле, $\operatorname{char} F \neq 2, H$ - алгебра обобщенных кватернионов $c$ делением над $F$. Тогда

$$
R(H)=8 .
$$


Таким образом, любая алгебра кватернионов с делением является алгеброй почти минимального ранга.

\section{8. Сложность умножения в алгебрах матриц}

В этом разделе мы улучшим оценку теоремы 13 на единицу в случае, когда рассматриваемая алгебра $A$ является алгеброй матриц над расширением базового поля $F$.

Лемма 5. Пусть $P\left(x_{1}, \ldots, x_{n}\right)$ - ненулевой полином степени $k$ над бесконечнылм полем $F$. Тогда существует набор $\alpha_{1}, \ldots, \alpha_{n}$, в котором не более $k$ ненулевых значений, на котором этот полином не равен 0 . Если $P$ существвенно зависит от переменной $x_{i}$, то одним из ненулевых значений можно взять $\alpha_{i}$.

Доказательство. Если полином $P$ не равен тождественно 0, то существует моном, входящий в него с ненулевым коэффициентом. В случае, если $P$ существенно зависит от $x_{i}$, существует моном с ненулевым коэффициентом, содержащий $x_{i}$. Так как степень полинома равна $k$, этот моном содержит не более $k$ переменных.

Подставим нули вместо всех переменных, кроме тех, которые входят в этот моном. Получим полином $P^{\prime}\left(x_{i_{1}}, \ldots, x_{i_{t}}\right), t \leqslant k$. Он содержит рассматриваемый моном, а потому не равен тождественно нулю, то есть найдутся значения $\alpha_{i_{1}}, \ldots, \alpha_{i_{t}}$, при подстановке которых полином принимает ненулевое значение. Если при этом рассматриваемый моном содержит переменную $x_{i}$, то $P^{\prime}$ можно записать в виде $\sum_{j=0}^{p} P_{j}^{\prime \prime}(\tilde{x}) x_{i}^{j}$, где $P_{j}^{\prime \prime}$ не зависят от $x_{i}$ и не все $P_{j}^{\prime \prime}$ при $j \geqslant 1$ тождественно равны нулю. Подставив вместо всех переменных, кроме $x_{i}$, значения так, что хотя бы один из $P_{j}^{\prime \prime}$ при $j \geqslant 1$ не обращается в 0 , получим, что $P^{\prime}$ существенно зависит от $x_{i}$ и можно подставить ненулевое значение вместо $x_{i}$ так, что получившееся значение многочлена $P^{\prime}$ будет ненулевым.

Лемма 6. Пусть $K$ - расширение бесконечного поля $F, \operatorname{dim}_{F} K=d, r: K \rightarrow F$ - ненулевой линейный функиионал, а $u_{1}, \ldots u_{n^{2} d}$ - некоторый базис $F$-алгебры $K^{n \times n}$. Многочлен

$$
Q(\tilde{x}, \tilde{y})=r\left(\operatorname{det}\left[\sum_{i=1}^{n^{2} d} x_{i} u_{i}, \sum_{i=1}^{n^{2} d} y_{i} u_{i}\right]\right) \in F[\tilde{x}, \tilde{y}]
$$

существенно зависит от всех переменных, кроме тех, которые соответствуют скалярным матрицам $u_{i}=\gamma I, \gamma \in K$, если такие элементы содержатся в рассматриваемом базисе.

Доказательство. Пусть $u_{1} \neq \gamma I$. Докажем, что многочлен $Q$ существенно зависит от $y_{1}$. Зависимость от остальных переменных доказывается аналогично. Так как $u_{1}-$ нескалярная матрица, найдется вектор $z_{1} \in K^{n}$ такой, что $u_{1} z_{1}=z_{2}$ не пропорционален $z_{1}$. Дополним пару $z_{1}, z_{2}$ до $K$-базиса пространства $K^{n}$ и будем рассматривать матрицы из $K^{n \times n}$ как операторы в этом базисе. В этом базисе $u_{1}$ имеет вид

$$
\left(\begin{array}{cccr}
0 & * & \ldots & * \\
1 & * & \ldots & * \\
\ldots & \ldots & \ldots & \ldots \\
0 & * & \ldots & *
\end{array}\right)
$$


где на местах, отмеченных звездочками, могут стоять произвольные значения из $K$. Выберем $x_{i}$ так, что

$$
X=\sum_{i=1}^{n^{2} d} x_{i} u_{i}
$$

в том же базисе имеет вид $\operatorname{diag}\left(\lambda_{1}, \lambda_{2}, \ldots, \lambda_{n}\right)$, где все $\lambda_{i}$ различны. При этом оператор ad $X: Y \mapsto[X, Y]$ переводит матрицу $Y=\left(y_{i j}\right)$ в матрицу

$$
[X, Y]=\left(\left(\lambda_{i}-\lambda_{j}\right) y_{i j}\right)
$$

Возьмем $\mu \in K$ такой, что $r(\mu) \neq 0$ и выберем матрицу

$$
Y=\sum_{i=1}^{n^{2} d} y_{i} u_{i}
$$

такую, что $Y z_{1}=0$, то есть

$$
Y=\left(\begin{array}{cccr}
0 & * & \ldots & * \\
0 & * & \ldots & * \\
\ldots \ldots & \ldots & \ldots \\
0 & * & \ldots & *
\end{array}\right),
$$

и $Y+u_{1}$ имеет вид

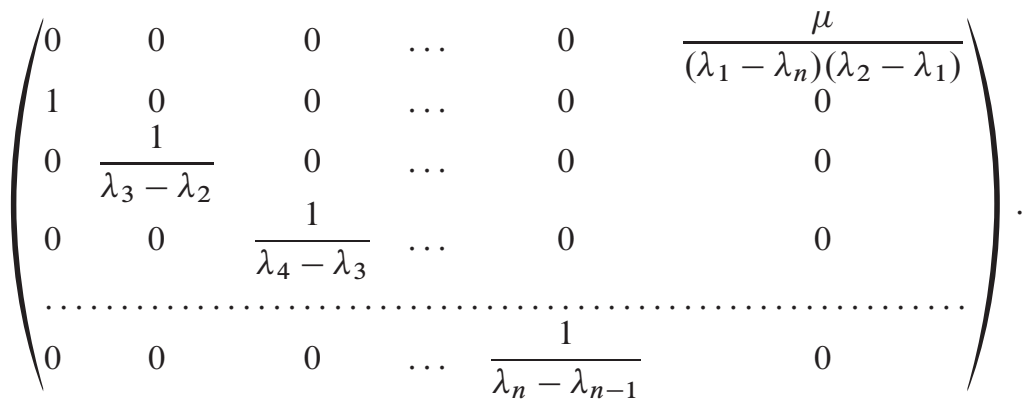

Тогда $\operatorname{det}[X, Y]=0$, так как первый столбец $[X, Y]$ будет нулевым, и

$$
\operatorname{det}\left[X, Y+u_{1}\right]=\mu \text {. }
$$

Получаем, что можно выбрать набор $(\tilde{x}, \tilde{y})$ так, что

$$
\begin{aligned}
Q(\tilde{x}, \tilde{y}) & =0, \\
Q\left(\tilde{x}, y_{1}+1, y_{2}, \ldots, y_{n^{2} d}\right) & \neq 0,
\end{aligned}
$$

что означает, что переменная $y_{1}$ является существенной.

Лемма 7. Пусть $K$ - расширение бесконечного поля $F, u n \geqslant 2$. Из любого базиса $F$-алгебры $K^{n \times n}$ можно выбрать не более $3 n-1$ элементов, в линейной оболочке которых найдутся три таких элемента $a_{1}, a_{2}, a_{3}$, что $a_{1} u\left[a_{1}^{-1} a_{2}, a_{1}^{-1} a_{3}\right]$ обратимы. 
Доказательство. Пусть $u_{1}, \ldots, u_{n^{2} d}$ - рассматриваемый базис. Если в нем есть обратимая матрица $u_{i}$, то возьмем $a_{1}=u_{i}$. Иначе пусть $r: K \rightarrow F-$ некоторый ненулевой линейный функционал и $\mu \in K$ таков, что $r(\mu) \neq 0$. Рассмотрим вначале многочлен

$$
P(\tilde{x})=r\left(\operatorname{det} \sum_{i=1}^{n^{2} d} x_{i} u_{i}\right) .
$$

Он ненулевой, так как существует матрица с определителем $\mu$, и является однородным многочленом степени $n$ : легко проверить, что

$$
P(\lambda \tilde{x})=\lambda^{n} P(\tilde{x}) .
$$

Согласно лемме 5, можно выбрать значения $x_{i}$, на которых $P$ не равен 0 , и среди которых $s \leqslant n$ ненулевых, то есть в линейной оболочке $s$ элементов базиса найдется невырожденная матрица $a_{1}$.

Далее, рассмотрим базис, составленный из элементов

$$
u_{i}^{\prime}=a_{1}^{-1} u_{i}
$$

и многочлен

$$
Q(\tilde{x}, \tilde{y})=r\left(\operatorname{det}\left[\sum_{i=1}^{n^{2} d} x_{i} u_{i}^{\prime}, \sum_{i=1}^{n^{2} d} y_{i} u_{i}^{\prime}\right]\right),
$$

исследованный в предыдущей лемме. Это однородный многочлен степени $2 n$. Снова применяя лемму 5 , получаем, что найдется $q \leqslant 2 n$ элементов нового базиса, содержащих матрицы

$$
a_{2}^{\prime}=a_{1}^{-1} a_{2}, \quad a_{3}^{\prime}=a_{1}^{-1} a_{3}
$$

такие, что $\left[a_{2}^{\prime}, a_{3}^{\prime}\right]$ обратима, причем один из элементов базиса может быть задан заранее, если он не является скалярной матрицей. Тогда в линейной оболочке соответствующих элементов исходного базиса содержатся матрицы $a_{2}$ и $a_{3}$.

В случае, когда в исходном базисе есть обратимая матрица $a_{1}=u_{i}$, мы получаем $2 n+1 \leqslant 3 n-1$ элементов, удовлетворяющих необходимым требованиям. В случае, когда все элементы базиса необратимы, мы в качестве одного из $q$ элементов, выбираемых на втором шаге, можем взять один из $s$ элементов, выбранных на первом, так как вырожденные матрицы не являются скалярными. В итоге получаем $s+q-1 \leqslant 3 n-1$ элементов, удовлетворяющих требуемым условиям.

Теорема 18. Пусть $K$ - расширение бесконечного поля $F, n \geqslant 2, A \cong K^{n \times n}$. Тогда

$$
R_{F}(A) \geqslant \frac{5}{2} \operatorname{dim}_{F} A-3 n+1 .
$$

Доказательство. Пусть $\sum_{k=1}^{r} f_{k} \otimes g_{k} \otimes w_{k}-$ билинейный алгоритм умножения в $A$. По лемме 1 , из набора $f_{k}$ можно выбрать базис пространства $A^{*}$. По лемме 7 , из двойственного базиса пространства $A$ можно выбрать не более $3 n-1$ элемента, в линейной оболочке которых найдутся элементы $a_{1}, a_{2}, a_{3}$ такие, что $a_{1}$ и $\left[a_{1}^{-1} a_{2}, a_{1}^{-1} a_{3}\right]$ обратимы.

Рассмотрим ограничение умножения на $\operatorname{lin}\left(a_{1}, a_{2}, a_{3}\right) \times A$. По лемме 11 , ранг этого билинейного отображения не меньше $(3 / 2) \operatorname{dim} A$. По лемме 2 , ранг исходного алгоритма не меньше $(\operatorname{dim} A-3 n+1)+(3 / 2) \operatorname{dim} A$, так как все $f_{k}$, кроме соответствующих выбранным $t \leqslant 3 n-1$ элементам, обращаются в 0 на $\operatorname{lin}\left(a_{1}, a_{2}, a_{3}\right)$. 
Следствие 5. Алгебра вида $K^{n \times n} n р и \operatorname{dim} K=2, n=3$ или $\operatorname{dim} K=3, n=2$ не является алгеброй почти минимального ранга.

Замечание 2. Условие бесконечности базового поля $F$ может быть опущено, так как из алгоритма для умножения в $K^{n \times n}$ над $F$, не увеличивая ранга, можно получить алгоритм умножения в $K(x)^{n \times n}$ над $F(x)$.

\section{9. Завершение классификации}

Из результатов раздела 5 следует, что любая полупростая алгебра почти минимального ранга является прямым произведением простых алгебр минимального ранга, то есть простых расширений базового поля и алгебр матриц $F^{2 \times 2}$, и не менее одной простой алгебры почти минимального ранга, то есть алгебры обобщенных кватернионов с делением. Докажем, что алгебра обобщенных кватернионов встречается ровно один раз.

Теорема 19. Если $H_{1}$ и $H_{2}$ - алгебры обобщенных кватернионов с делением, и

$$
A=H_{1} \times H_{2},
$$

mo

$$
R(A)=16 .
$$

Доказательство. Верхняя оценка $R(A) \leqslant 16$ следует из теоремы 17 и свойств ранга. Докажем нижнюю оценку. В тексте доказательства будем отождествлять $H_{1}$ и $H_{2}$ с их образами в $A$.

Пусть $\sum_{k=1}^{r} f_{k} \otimes g_{k} \otimes z_{k}-$ билинейный алгоритм для умножения в $A$. Элементы $f_{k}$ порождают все пространство $A^{*}$. Если каждый функционал $f_{k}$ равен нулю либо на $H_{1}$, либо на $H_{2}$, то алгоритм распадается на сумму двух алгоритмов для алгебр $H_{1}$ и $H_{2}$, сложность каждого из которых равна 8.

В противном случае найдется базис, состоящий из $f_{k}$, такой, что в двойственном базисе есть элемент вида $a=\left(a^{\prime}, a^{\prime \prime}\right)$ с ненулевыми $a^{\prime}$ и $a^{\prime \prime}$. Пусть, без ограничения общности, $f_{1}, \ldots, f_{8}$ - такой базис, $u_{1}, \ldots, u_{8}-$ двойственный базис, и пусть $u_{1}$ имеет две ненулевые компоненты. В базисе $u_{1}, \ldots, u_{8}$ найдется такой элемент $u_{i}=\left(u_{i}^{\prime}, u_{i}^{\prime \prime}\right)$, что $\left(u_{1}^{\prime}\right)^{-1} u_{i}^{\prime} \notin F$. Пусть, без ограничения общности, этот элемент есть $u_{2}$.

Убрав из алгоритма слагаемые с индексами $3 \leqslant k \leqslant 8$ и ограничив все $f_{k}$ на $\operatorname{lin}\left(\left\{u_{1}, u_{2}\right\}\right)$, мы получим алгоритм $\sum_{k=1}^{r-6} f_{k}^{\prime} \otimes g_{k}^{\prime} \otimes z_{k}^{\prime}$ для отображения, полученного ограничением умножения на $\operatorname{lin}\left(\left\{u_{1}, u_{2}\right\}\right) \times A$, где $f_{1}^{\prime}, f_{2}^{\prime}-$ двойственный базис к $u_{1}, u_{2}$.

Докажем, что сложность этого алгоритма не меньше 10. Допустим, она равна 9. Так как $u_{1} v \neq 0$ для любого $v \in A$, функционалы $g_{k}^{\prime}$ при $k=1,3, \ldots, 9$ образуют базис $A^{*}$. Пусть $v_{1}, v_{3} \ldots, v_{9}-$ двойственный базис. Тогда

$$
\begin{aligned}
& u_{1} v_{j}=f_{j}^{\prime}\left(u_{1}\right) z_{j}, \\
& u_{2} v_{j}=f_{j}^{\prime}\left(u_{2}\right) z_{j}+g_{2}^{\prime}\left(v_{j}\right) z_{2} .
\end{aligned}
$$

Так как $v_{i}-$ базис и $g_{2}^{\prime} \neq 0$, найдется такой индекс $j^{*} \in\{1,3, \ldots, 9\}$, что $g_{2}^{\prime}\left(v_{j *}\right) \neq 0$.

Исключая $z_{2}$ из соотношений (29), получаем, что $u_{1}\left(v_{j}-v_{j} * g_{2}^{\prime}\left(v_{j}\right) / g_{2}^{\prime}\left(v_{j} *\right)\right)$ и $u_{2}\left(v_{j}-v_{j} g_{2}^{\prime}\left(v_{j}\right) / g_{2}^{\prime}\left(v_{j *}\right)\right)$ пропорциональны друг другу, то есть существуют семь линейно независимых векторов

$$
x_{j}=v_{j}-\frac{g_{2}^{\prime}\left(v_{j}\right)}{g_{2}^{\prime}\left(v_{j^{*}}\right)} v_{j^{*}},
$$


где $j \neq j^{*}$, для которых $u_{1} x_{j}$ пропорционально $u_{2} x_{j}$. Однако, если $x=\left(x^{\prime}, x^{\prime \prime}\right)$ и $x^{\prime} \neq 0$, то из пропорциональности $u_{1}^{\prime} x^{\prime}$ и $u_{2}^{\prime} x^{\prime}$ следует пропорциональность $\left(u_{1}^{\prime}\right)^{-1} u_{2}$ и 1 , что противоречит выбору $u_{2}$. Значит, все семь линейно независимых $x_{j}$ имеют нулевую первую компоненту. Получили противоречие, доказывающее теорему.

Отсюда следует, что в разложении алгебры почти минимального ранга в прямое произведение простых алгебр не может быть двух алгебр кватернионов с делением, поскольку

$$
\Delta\left(H_{1} \times H_{2}\right)=2 \text {. }
$$

В итоге получаем окончательную классификацию, приведенную в следующей теореме.

Теорема 20. Любая полупростая алгебра почти минимального ранга над бесконечным полем $F$, char $F \neq 2$, имеет вид $H$ или $H \times B$, где $H$-алгебра обобщенных кватернионов с делением, $B$ - полупростая алгебра минимального ранга.

\section{0. О классификации над другими полями}

Все результаты этой работы, кроме теоремы о ранге алгебр обобщенных кватернионов, справедливы над произвольными полями. Для классификации полупростых алгебр почти минимального ранга над бесконечным полем характеристики 2 необходимо доказать или опровергнуть аналогичный результат в этом случае. Также необходимо рассмотреть случай непростого несепарабельного расширения, например, $F(x, y)$ над $F\left(x^{2}, y^{2}\right)$. Над полями характеристики 2 алгебры обобщенных кватернионов имеют структуру, описанную в следующей теореме.

Теорема 21 ([6]). Если char $F=2$, то любая алгебра обобщенных кватернионов порождается двумя элементами $i$ и $j$, удовлетворяющими условиям

$$
\begin{aligned}
i(i+1) & =p, \\
j^{2} & =q, \\
i j & =j(i+1),
\end{aligned}
$$

где $p, q \in F, q \neq 0$. Элементы $1, i, j, k=i j$ образуют базис алгебры.

Для классификации над конечными полями основной проблемой является определение ранга расширений, степень которых слишком велика для использования алгоритмов типа «вычисление-интерполяция». Данная проблема активно изучается с помощью методов алгебраической геометрии (см., например, [11]).

\section{Список литературы}

1. Alder A., Strassen V., On the algorithmic complexity of associative algebras. Theor. Comput. Sci. (1981) 15, 201-211.

2. Bläser M., A complete characterization of the algebras of minimal bilinear complexity. SIAM J. Comput. (2004) 34, 277-298.

3. Bläser M., de Voltaire A. M., Semisimple algebras of almost minimal rank over the reals. Theor. Comput. Sci. (2009) 410, 5202-5214.

4. Ван дер Варден Б. Л., Алгебра. Наука, Москва, 1976. 
5. Дрозд Ю. А., Кириченко В. В., Конечномерные алгебры. Вища школа, Киев, 1980.

6. Knus M. A., Merkurjev A., Rost M., et al., The book of involutions. AMS, Providence, 1998.

7. Bürgisser P., Clausen M., Shokrollahi M. A., Algebraic complexity theory. Springer, Berlin, 1997.

8. Strassen V., Rank and optimal computation of generic tensors. Linear Algebra Appl. (1983) 52-53, 645-685.

9. Bläser M., Beyond the Alder-Strassen bound. Theor. Comput. Sci. (2005) 331, 3-21.

10. Bläser M., On the complexity of the multiplication of matrices of small formats. J. Complexity (2003) 19, 43-60.

11. Ballet S., An improvement of the construction of the D. V. and G. V. Chudnovsky algorithm for multiplication in finite fields. Theor. Comput. Sci. (2006) 352, 293-305.

Статья поступила 9.11.2012. 